Journal for ImmunoTherapy of Cancer

\title{
Involvement of the M-CSF/IL-34/CSF- 1R pathway in malignant pleural mesothelioma
}

To cite: Blondy T, d'Almeida SM, Briolay T, et al. Involvement of the M-CSF/L-34/CSF-1R pathway in malignant pleural mesothelioma. Journal for ImmunoTherapy of Cancer 2020;8:e000182. doi:10.1136/ jitc-2019-000182

- Additional material is published online only. To view please visit the journal online (http://dx.doi.org/10.1136/jitc2019-000182).

Accepted 08 May 2020

Check for updates

(C) Author(s) (or their employer(s)) 2020. Re-use permitted under CC BY-NC. No commercial re-use. See rights and permissions. Published by BMJ.

For numbered affiliations see end of article.

\section{Correspondence to}

Dr Christophe Blanquart;

christophe.blanquart@inserm.fr

\section{ABSTRACT}

Background Malignant pleural mesothelioma (MPM) is a rare and aggressive cancer related to asbestos exposure. The tumor microenvironment content, particularly the presence of macrophages, was described as crucial for the development of the disease. This work aimed at studying the involvement of the M-CSF (CSF-1)/IL-34/CSF-1R pathway in the formation of macrophages in MPM, using samples from patients.

Methods Pleural effusions (PEs), frozen tumors, primary MPM cells and MPM cell lines used in this study belong to biocollections associated with clinical databases. Cytokine expressions were studied using real-time PCR and ELISA. The Cancer Genome Atlas database was used to confirm our results on an independent cohort. An original threedimensional (3D) coculture model including MPM cells, monocytes from healthy donors and a tumor antigenspecific cytotoxic CD8 T cell clone was used.

Results We observed that high interleukin (IL)-34 levels in PE were significantly associated with a shorter survival of patients. In tumors, expression of CSF1 was correlated with 'M2-like macrophages' markers, whereas this was not the case with IL34 expression, suggesting two distinct modes of action of these cytokines. Expression of IL34 was higher in MPM cells compared with primary mesothelial cells. Particularly, high expression of IL34 was observed in MPM cells with an alteration of CDKN2A. Finally, using 3D coculture model, we demonstrated the direct involvement of MPM cells in the formation of immunosuppressive macrophages, through activation of the colony stimulating factor-1 receptor (CSF1-R) pathway, causing the inhibition of cytotoxicity of tumor antigen-specific $\mathrm{CD}^{+} \mathrm{T}$ cells. Conclusions The M-CSF/IL-34/CSF-1R pathway seems strongly implicated in MPM and could constitute a therapeutic target to act on immunosuppression and to support immunotherapeutic strategies.

\section{BACKGROUND}

Malignant pleural mesothelioma (MPM) is a particularly aggressive disease related to asbestos exposure with a median survival lower than 1 year. The therapeutic options for this pathology are very limited, and the firstline regimen, which consists of a combination of cisplatin and pemetrexed, only increases survival by approximately 3 months. ${ }^{1}$ Therefore, new therapeutic strategies are required to improve the outcome of the disease.

Recently, several interesting studies have described the cellular content of the mesothelioma microenvironment, ${ }^{2}{ }^{3}$ among which macrophages seem particularly interesting. These cells can exist on two opposite phenotypes, M1 macrophages which have pro-inflammatory and anti-tumor properties and M2 macrophages which have immunosuppressive and tumor-promoting characteristics. ${ }^{4}$ Macrophages are highly plastic cells and therefore these two extreme phenotypes are rarely observed in a physiological context. Indeed, depending on the environment, macrophages will be defined as 'M1like' or 'M2-like' macrophages based on the differential expression of a set of markers. ${ }^{4}$ Several studies have shown that the presence of M2-like macrophages in mesothelioma tumors has a poor prognosis. ${ }^{356}$

One pathway of differentiation of monocytes into macrophages requires activation via the colony stimulating factor-1 receptor (CSF-1R). CSF-1R (CD115) is a tyrosine kinase receptor leading to activation of the PI3K/Akt and MAPK pathways following ligand binding. Recently, we demonstrated that pleural effusions (PEs) from patients with MPM led monocytes to differentiate into M2-like macrophages, in a CSF-1R-mediated manner. Moreover, we observed the presence of M-CSF (CSF-1) in PE from patients with MPM and we described that M2 macrophages decreased the efficacy of the cisplatinpemetrexed treatment in MPM. ${ }^{7}$ Based on our findings, the CSF-1R pathway could thus be considered as a therapeutic target for reducing local immunosuppression. Two cytokines have been described as ligands for CSF1R: M-CSF (CSF1) and IL-34 (IL34) ${ }^{8}$ which 
have already been described to be involved in different cancers. ${ }^{9}$ However, in mesothelioma, there are few data available on the expression of M-CSF in the tumor microenvironment and no data regarding interleukin (IL)-34.

As these two cytokines are able to activate the CSF-1R transduction pathway in monocytes and to induce M2 differentiation, ${ }^{10}$ in this study we aimed at evaluating the presence of M-CSF and IL-34 in MPM, the capacity of MPM cells to induce M2-like macrophages and finally the consequences of the presence of these macrophages on the functionality of immune effector cells. We first measured cytokine levels in our collection of PE from patients. We then determined the mRNA expression of both CSF1 and IL34 in MPM tumors, MPM primary cells and MPM cell lines. Finally, using a model of coculture in three dimensions with mesothelioma cells and monocytes, we analyzed the phenotype of macrophages and the impact on the cytotoxic activity of $\mathrm{CD} 8^{+} \mathrm{T}$ cells.

\section{METHODS}

\section{Collection of mesothelioma cell lines and pleural effusions}

The mesothelioma and other neoplasia cell lines were established from pleural fluids of patients in our laboratory. ${ }^{11}$ All cell lines were maintained in RPMI-1640 medium (Gibco) supplemented with $2 \mathrm{mM} \mathrm{L-glutamine,}$ $100 \mathrm{IU} / \mathrm{mL}$ penicillin, $0.1 \mathrm{mg} / \mathrm{mL}$ streptomycin and $10 \%$ heat-inactivated fetal calf serum (FCS) (Gibco) and cultured at $37^{\circ} \mathrm{C}$ in a $5 \% \mathrm{CO}_{2}$ atmosphere. The primary peritoneal mesothelial cells, MES-F, were purchased from Tebu-bio biosciences and cultured according to the manufacturer's recommendations. Meso 34 NanoLuc cells were obtained after transfection of Meso 34 cells with pNL2.1[Nluc/Hygro] (Promega). After 48 hours, selection was performed using hygromycine (Invitrogen) $(125 \mu \mathrm{g} / \mathrm{mL})$ for 2 weeks. Expression of NanoLuc was assessed by seeding cells at $5 \times 10^{3}$ cells per well of whitewalled 96-well plate (Corning). Twenty-four hours later, after a wash with phosphate-buffered saline (PBS), coelenterazine $(3.5 \mu \mathrm{M})$ (Interchim) was added and the luminescence signal was recorded after $10 \mathrm{~min}$ for $1 \mathrm{~s}$ using a Mithras LB 940 microplate analyzer (Berthold Technologies).

MPM primary cell lines were established at "Functional Genomics of Solid Tumors" laboratory, Paris, from surgical resections, pleural biopsies, or malignant pleural fluids of confirmed MPM cases, obtained from several French hospitals with patient's consents. Most of them were used in several previous studies showing their relevance to MPM primary tumors. Genetic alterations in key genes of mesothelial carcinogenesis $(C D K N 2 A, B A P 1, N F 2$, LATS2 and TP53) and C1/C2 subtypes of the molecular classification were determined in this MPM series. ${ }^{12}{ }^{13}$ Normal mesothelial cells were cultured from surgical resection of blebs from patients with spontaneous pneumothoraxes. Gene expressions were determined using cultures at lowpassage number.
PEs from patients with a suspected mesothelioma were aseptically collected by thoracocentesis at the Laënnec Hospital (St-Herblain, France) between 1998 and 2016. Samples were centrifuged at $1000 \times \mathrm{g}$ in a Heraeus Multifuge for $20 \mathrm{~min}$ at $+4^{\circ} \mathrm{C}$ and supernatants were aliquoted and stored at $-80^{\circ} \mathrm{C}$. Serum samples were also collected at the Laënnec Hospital, aliquoted and stored at $-80^{\circ} \mathrm{C}$. Diagnoses were established by both fluid cytology and immunohistochemical staining of pleural biopsies performed by the pathology department at Laënnec Hospital (St-Herblain, France) and then externally confirmed by Mesopath, the French panel of pathology experts for the diagnosis of mesothelioma. All recruited patients had received no prior anticancer therapy and gave signed informed consent. All the collected samples and the associated clinical information were registered in a database (DC-2011-1399) validated by the French ministry of research.

The collection of MPM tumor samples and normal pleura was previously described. ${ }^{14}$ Transcriptome microarray data were available from 63 tumor samples of the same collection ${ }^{15}$; ArrayExpress database: accession codes E-MTAB-6877.

\section{Cytokine quantification}

IL-34 and M-CSF titrations were performed with the Human IL-34 DuoSet ELISA and the Human M-CSF Quantikine ELISA kit (both from R\&D Systems), respectively, following the manufacturers' recommendations. The supernatants of MCTS were aliquoted and stored at $-80^{\circ} \mathrm{C}$ until use. Cytokines were measured using the LEGENDplex Human M1/M2 macrophage panel (BioLegend) according to the manufacturer's recommendations.

\section{RNA isolation and real-time PCR from cell lines}

Total RNA was isolated using the Nucleospin RNAII Kit according to the manufacturer's protocol (MachereyNagel). One microgram of total RNA was reversetranscribed using Moloney murine leukemia virus reverse transcriptase (Invitrogen). Real-time PCR (RT-PCR) was carried out using an Mx3005P thermocycler (Stratagene). PCR was performed using QuantiTect Primer Assays (Qiagen) and the $\mathrm{RT}^{2}$ Real-Time SYBR-Green/ROX PCR Mastermix (Qiagen), according to the manufacturer's instructions. The relative amount of the target RNA was determined using the MxPro software, by comparison with the corresponding standard curve for each sample performed in duplicate. Each transcript level was normalized by division with the expression values of the acidic ribosomal phosphoprotein $\mathrm{P} 0$ housekeeping gene $(R P L P O)$, used as an internal standard.

For gene expression analysis on MPM primary cell lines and frozen tumor samples, total RNA was isolated using Trizol (Thermo Fisher Scientific) or AllPrep DNA/ RNA/miRNA Universal kit (Qiagen) according to the manufacturer's protocol. $1.5 \mu \mathrm{g}$ of total RNA was reversetranscribed using High-Capacity cDNA Reverse Transcription Kit (Thermo Fisher Scientific). RT-PCR was carried 
out using ABI Prism 7900HT Real-Time PCR System. PCR was performed using Taqman assays (Thermo Fisher Scientific) according to the manufacturer's instructions. IL34 and CSF1 transcript levels were normalized by the mean of the expression values of the five housekeeping genes Ribosomal 18S, ACTB, CLTC, GAPDH and TBP $(-\Delta \mathrm{Ct})$. The following Taqman assays have been used: IL34 (Hs01050926_m1), CSF1 (Hs00174164_m1), $18 S$ (Hs03928990_g1), ACTB (Hs01060665_g1), CLTC (Hs00964504_m1), GAPDH (Hs02758991_g1) and TBP (Hs00427620_m1).

\section{Analysis of The Cancer Genome Atlas dataset}

All RNAseqv2 samples from the The Cancer Genome Atlas (TCGA)-MESO dataset ( $\mathrm{n}=87$ patients) are available on the Broad's Genome Data Analysis Center (http:// gdac.broadinstitute.org/). Gene expressions as RNA-seq by expectation maximization values (RSEM values) were analyzed. Clinical data for these samples were downloaded from FireBrowse (http://firebrowse.org; version 2018_02_26 for MESO).

\section{Multicellular tumor spheroid formations}

Meso 34 cells were mixed with or without monocytes from healthy donors obtained by elutriation (DTC Core Facility, Nantes Hospital) ${ }^{16}$ at a ratio of 2:1 in 96-well U bottom plates NUNCLON SPHERA (Thermo Fisher Scientific) and in a volume of $180 \mu \mathrm{L}$ of complete culture medium. The plates were centrifuged $2 \mathrm{~min}$ at $800 \times \mathrm{g}$ and incubated at $37^{\circ} \mathrm{C}$ in a $5 \% \mathrm{CO}_{2}$ atmosphere for 3 days.

\section{Immunohistochemistry on multicellular tumor spheroids}

Multicellular tumor spheroids (MCTSs), constituted at formation of $20 \times 10^{3}$ cells, were fixed with $4 \%$ paraformaldehyde (Electron Microscopy Sciences) for 24 hours at room temperature (RT). After one wash in PBS, MCTSs were included in HistoGel (Microtech, Thermo Fisher Scientific). Then, immunohistochemical analysis was performed using standard techniques by the Cellular and Tissue Imaging Core Facility of Nantes University (MicroPICell). The anti-CD163 antibody (Invitrogen) was used at 1/100 and the anti-CD14 antibody (Abcam) was used at $0.5 \mu \mathrm{g} / \mathrm{mL}$. The revelation was performed using Leica Bond Polymer Refine Detection (Leica). Pictures were obtained using a NanoZoomer 2.0HT (Hamamatsu).

\section{Confocal microscopy}

MCTSs, constituted of $20 \times 10^{3}$ cells, were collected, washed one time in PBS and fixed in paraformaldehyde 4\% (Electron Microscopy Sciences) for 48 hours at RT. MCTSs were washed once with PBS and permeabilized for 24 hours with PBS containing 2\% Triton X-100 at RT. This solution was removed and then MCTSs were incubated with a solution of PBS containing 1\% BSA, 0.2\% Triton X-100, Hoescht $5 \mu \mathrm{g} / \mathrm{mL}$ (Sigma-Aldrich) and anti-CD163-Alexa Fluor 647 (BD Biosciences) for 48 hours at $4^{\circ} \mathrm{C}$. Two steps of washing were performed with PBS containing 3\% $\mathrm{NaCl}$ and $0.2 \%$ Triton X-100 for 2 hours at RT. Finally, MCTSs were resuspended into a RapiClear solution (SunJin Lab) and observed with a confocal microscope (Nikon A1R Si).

\section{Flow cytometry}

MCTSs ( $n=24)$ were collected, washed once in PBS and incubated with Trypsin $0.05 \%$ EDTA (Gibco) for approximately $10 \mathrm{~min}$. Each $3 \mathrm{~min}$, trypsin was removed and diluted in the culture medium to preserve detached cells and new trypsin was added on the residual MCTS to optimize dissociation of MPM cells and macrophages. The cell suspension was centrifuged at $800 \times \mathrm{g}$ for $60 \mathrm{~s}$ in an Eppendorf Minispin. Cells were washed with PBS and stained with an anti-CD14-PE (clone REA-599, Miltenyi Biotec), an anti-CCR2-BV605 (clone K036C2, Biolegend), an antiHLA-DR-FITC (clone G46-6, BD Pharmingen) and an anti-CD163-alexa647 (clone GH/61, BD Pharmingen), in RPMI-1640 with $10 \% \mathrm{FCS}$ for $30 \mathrm{~min}$ at $4^{\circ} \mathrm{C}$. IgG1 ${ }_{\mathrm{K}}$-FITC (clone MOPC-21, BD Pharmingen), IgG2a-BV605 (clone MOPC-173, Biolegend), REA control-PE (clone REA293, Miltenyi Biotec) and IgG1 $_{\mathrm{K}}$-alexa647 (clone MOPC-21, BD Pharmingen) isotypes were used as controls. Samples were washed twice and then resuspended in PBS. Sample acquisition was performed using an LSR-Fortessa X-20 cytometer (Becton Dickinson). Results were analyzed with the DIVA Software (Tree Star).

\section{T cell clone cytotoxicity assay}

Meso 34 NanoLuc MCTSs, constituted of $5 \times 10^{3}$ cells including $30 \%$ of monocytes, were obtained as described above. Then, $20 \times 10^{3}$ cells of the previously described HLA-A*0201/MUC1(950-958)-specific CD8+ Tcell clones were added. ${ }^{17}$ Cytotoxicity of the CD8 T cell clone toward Meso 34 NanoLuc cells was evaluated by measuring nanoluciferase activity released in MCTS supernatants following cell lysis as follows. After 24 hours, $45 \mu \mathrm{L}$ of medium was collected and light emission at $480 \mathrm{~nm}$ was measured immediately after addition of $5 \mu \mathrm{L}$

Table 1 Description of groups and demographic characteristics of recruited patients for pleural effusion study

\begin{tabular}{llll}
\hline & MPM & $\begin{array}{l}\text { Other } \\
\text { neoplasia }\end{array}$ & BPE \\
\hline Description & $\begin{array}{l}96 \\
76 \text { epithelioid } \\
7 \text { sarcomatoid } \\
\text { 8 biphasic }\end{array}$ & $\begin{array}{l}105 \\
60 \text { lung } \\
4 \text { others }\end{array}$ & \\
& & & \\
& 5 unspecified & & \\
& $68.8 \pm 9.6$ & $64.3 \pm 16.6$ & $74.1 \pm 11.5$ \\
$\begin{array}{l}\text { Age, years } \\
\text { (mean } \pm \text { SD) }\end{array}$ & & & \\
Male sex, (\%) & 83.3 & 54.3 & 84.6 \\
$\begin{array}{l}\text { Confirmed } \\
\text { asbestos } \\
\text { exposure (\%) }\end{array}$ & 68.7 & 15.2 & 30.7 \\
\hline
\end{tabular}

MPM, malignant pleural mesothelioma ; BPE, benign pleural effusion 
of coelenterazine at $30 \mu \mathrm{M}$ using Mithras LB 940 microplate analyzer (Berthold Technologies).

\section{Data and statistical analyses}

The estimation of the abundance of immune cell populations infiltrating MPM was done by using Microenvironment Cell Population Counter (MCP-counter) software on the gene expression dataset. ${ }^{15-18}$ Comparisons were performed using parametric paired t-test or KruskalWallis test followed by the Dunn's post hoc test. Log-rank Mantel-Cox test was used for survival analyses. Correlations were evaluated using non-parametric Spearman test. All statistical analyses were performed using GraphPad Prism (Prism V.6 for Windows) except univariate and multivariate Cox regression analysis that was performed using R statistical software.

\section{RESULTS}

\section{Expression of IL-34 and M-CSF in pleural effusions from} patients with MPM and prognostic value

In order to evaluate the involvement of M-CSF and IL-34 in MPM, we measured the expression of these cytokines in our collection of PE from patients. This collection is constituted of 96 MPM, 105 other neoplasia and 26 benign pleural effusions (BPEs) (table 1).

First, we observed that all the effusion types tested contained detectable level of M-CSF (figure 1A). There was no significant difference in the expression of M-CSF between MPM, other neoplasia and BPE groups. There was also no significant difference between malignant and benign PE (see online supplementary figure S1A). When considering subgroups, sarcomatoid mesothelioma (SM) seemed to express more M-CSF than the others (see online supplementary figure S1B). Regarding IL-34, MPM and other neoplasia groups had a higher expression level compared with BPE. Expression of IL-34 was significantly higher in malignant $\mathrm{PE}$ compared with BPE (see online supplementary figure S1C). However, a strong dispersion in the values of IL-34 was observed inside groups and subgroups (figure $1 \mathrm{~B}$ and see online supplementary figure S1C), particularly for malignant PE. Indeed, less than $50 \%$ of the samples were positive for IL-34. Interestingly, the SM subgroup presented $71.4 \%$ of positive samples, whereas the other subgroups presented less than $50 \%$ of positive samples (figure 1C). Figure 1D shows that, in MPM samples, M-CSF levels were significantly higher in IL-34 positive PE compared with IL-34 negative PE.

Then, we evaluated the prognostic value of M-CSF and IL-34 in PE of patients with MPM. Overall survival data were available for 74 patients and global median survival for patients with MPM was 349 days. Patients were separated in two groups according to the M-CSF mean values and according to positivity for IL-34. Patients with M-CSF levels above mean presented a significant lower survival than the others (238 days vs 387 days; $p=0.0395$ ) (figure 1E). Patients with IL-34 positive PE presented
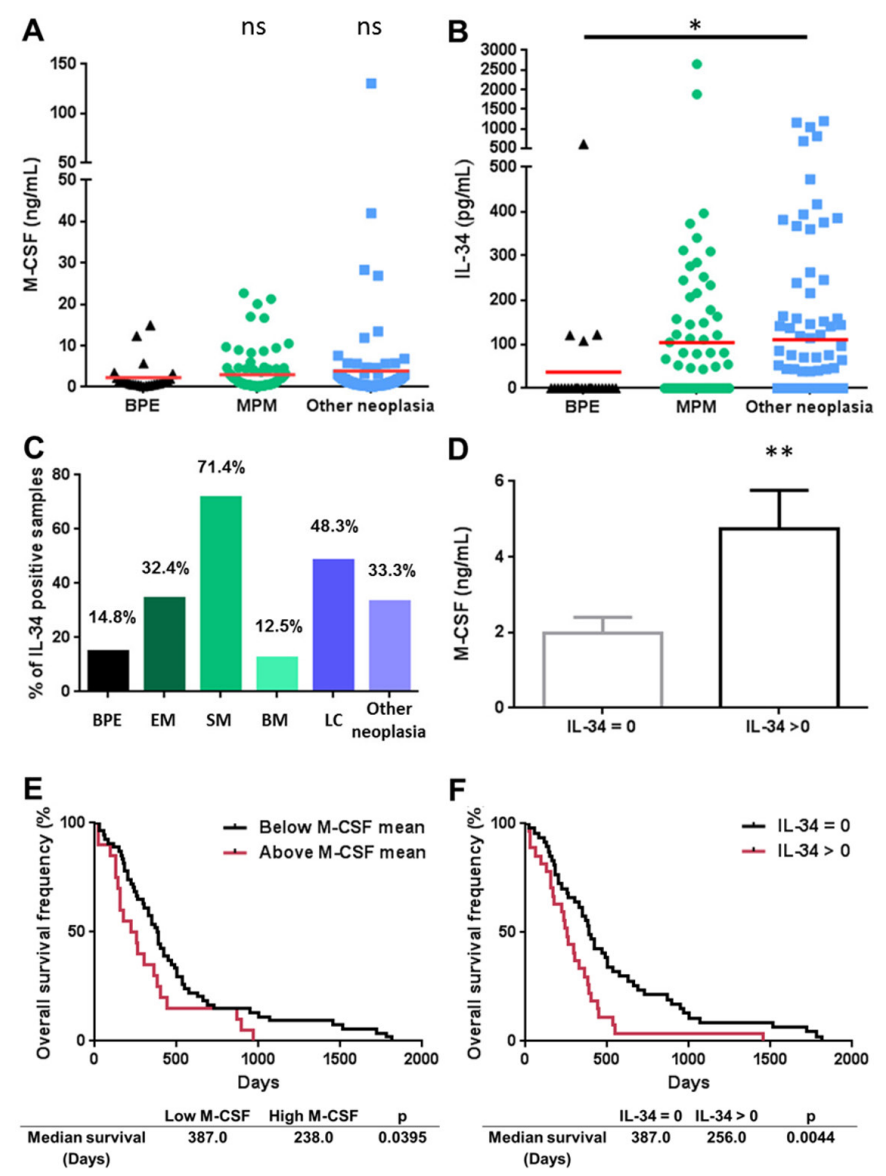

Figure 1 Expression of M-CSF and IL-34 in pleural effusions from patients and prognostic value. (A) M-CSF and (B) IL-34 expression in pleural effusions from patients with MPM $(n=96)$, other neoplasia $(n=105)$ or BPE $(n=26)$. Red bars correspond to means. (C) Percentage of IL-34 positive samples in the different groups of patients. (D) Levels of MCSF in IL-34 negative and positive samples. Patients were split in 'high expression' and 'low expression' groups based on the mean of expression of M-CSF (E) or on positive and negative expression of IL-34 (F) in MPM pleural effusions, and differences in survival between the two groups were assessed using log-rank tests. ${ }^{*} p<0.05$; ${ }^{* *} p<0.01$. BM, biphasic mesothelioma; BPE, benign pleural effusion; EM, epithelioid mesothelioma; LC, lung cancer; MPM, malignant pleural mesothelioma; SM, sarcomatoid mesothelioma.

a significant lower survival than the others (256 days vs 387 days; $p=0.0044$ ) (figure $1 \mathrm{~F}$ ). A multivariate analysis was performed including histologic subtype, IL-34 and M-CSF as parameters. We found IL-34 score to be an independent prognostic factor in our MPM cohort (online supplementary figure $\mathrm{S} 2$ ), whereas histologic subtype and M-CSF did not reach significance $(\mathrm{p}=0.243$ and $\mathrm{p}=0.218$, respectively).

\section{Expression of CSF1 and IL34 in MPM tumors and in primary MPM cells}

To complete our study, we analyzed the mRNA expressions of CSFI and IL34 in a collection of MPM tumors $(\mathrm{n}=178)$ and normal pleura $(\mathrm{n}=26)$ biopsies. A slightly lower level of CSF1 gene expression was observed in MPM 
A

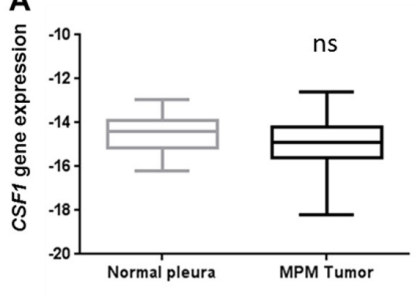

B
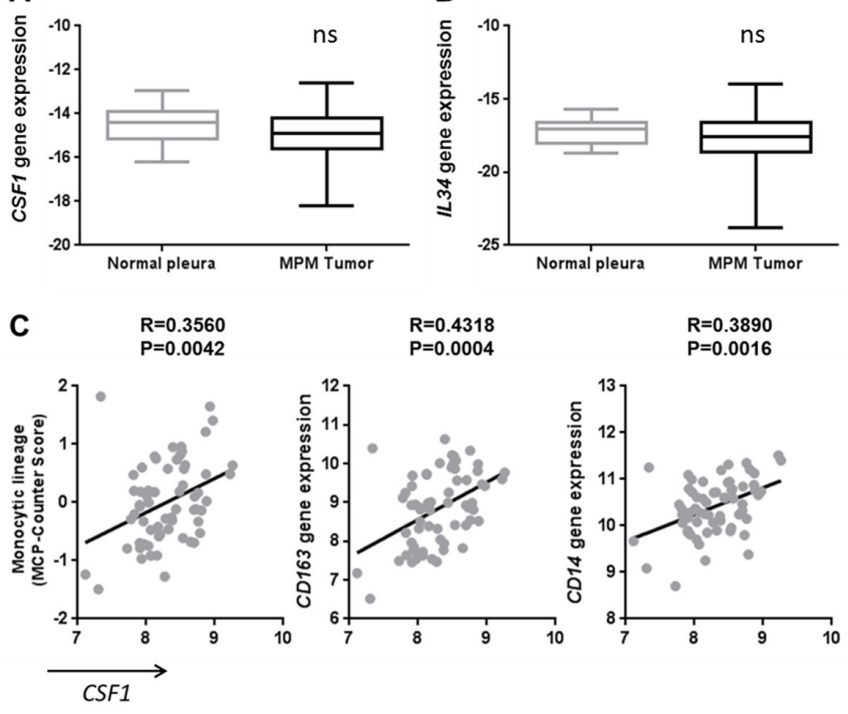

$R=0.4318$
$P=0.0004$
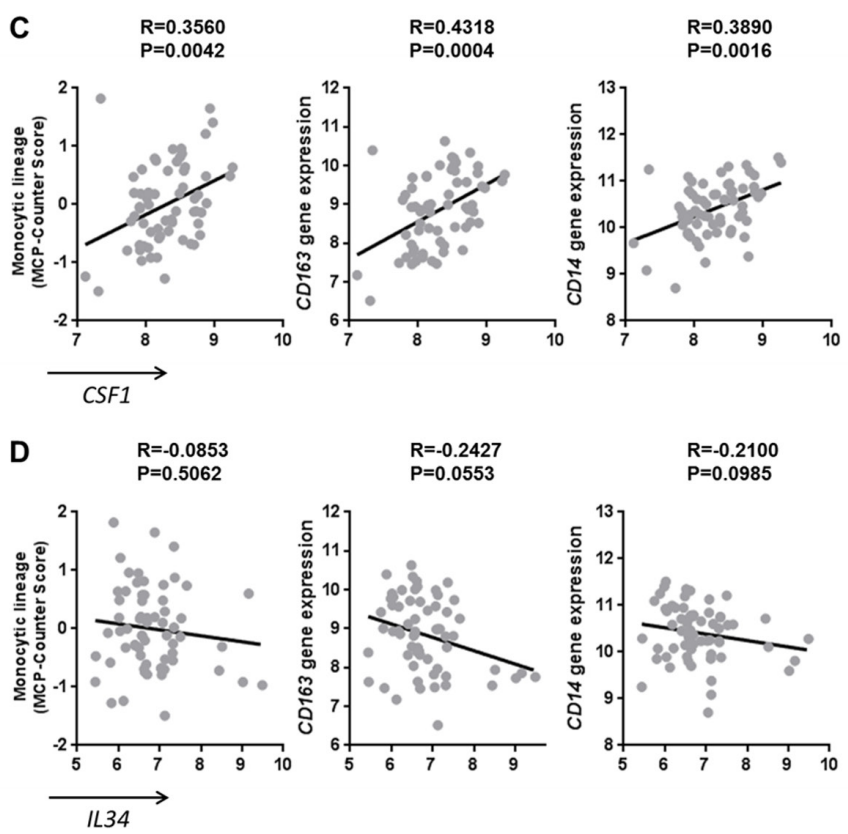

Figure 2 Correlation of CSF1 expression with tumorassociated macrophage markers in malignant pleural mesothelioma (MPM) tumors. (A) CSF1 and (B) IL34 gene expressions measured using quantitative real-time PCR in MPM tumors $(n=178)$ and normal pleura $(n=26)$. (C-D) Correlation between CSF1 (C) or IL34 (D) expressions and monocytic lineage-specific $C D 163$ and $C D 14$ expressions using transcriptomic data of MPM tumor samples $(n=63)$.

tumors compared with normal pleura $(\mathrm{p}=0.0456)$ but no difference in the IL34 gene expression (figure 2A,B, respectively). By performing a correlation study using transcriptomic data obtained on 63 samples of the previous MPM tumor biopsy collection, we observed that CSF1 expression was positively associated with the expression of its receptor CSF1R, whereas it was not the case with IL34 expression (see online supplementary figure S3A and B). Moreover, CSF1 expression was associated with the infiltration of the tumor by immune cells of the monocytic lineage determined by the MCP-Counter tool $^{18}$ on transcriptomic data (figure $2 \mathrm{C}$ ). More precisely, CSF1 expression was correlated with the expression of tumor-associated macrophage markers, particularly of the 'M2-like' macrophage subset, such as CD163 and CD14 (figure 2C), and with a tendency for IL1O (data not shown). These correlations were not observed with the expression of IL34 (figure 2D). These results were confirmed using data from the TCGA database and a positive correlation was observed between $C S F 1$ expression
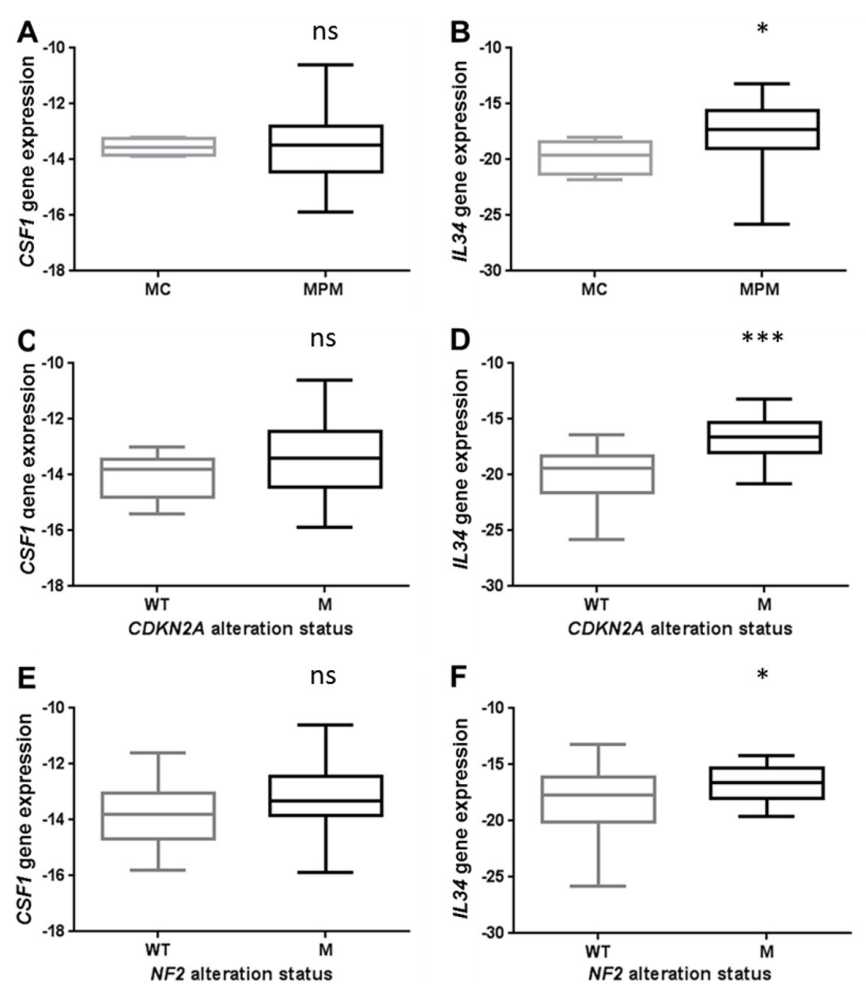

Figure 3 Expression of CSF1 and IL34 in MPM cells. (A) CSF1 and (B) IL34 gene expressions measured using quantitative real-time PCR (qRT-PCR) in primary malignant pleural mesothelioma (MPM) cells $(n=69)$ and normal mesothelial cells ( $n=4 ; M C)$. (C) CSF1 and (D) IL34 gene expressions measured using qRT-PCR in primary MPM cells with or without $C D K N 2 A$ genetic alteration. $(E) C S F 1$ and $(F)$ IL34 gene expressions measured using qRT-PCR in primary MPM cells with or without NF2 genetic alteration. ${ }^{*} p<0.05$; ${ }^{\star * *} \mathrm{p}<0.001$.

and IL10 (see online supplementary figure S3C-D, table S1 and online supplementary figure S4).

In order to focus only on malignant cells, we measured the mRNA expression of $C S F 1$ and $I L 34$ in a collection of samples of primary MPM cells $(n=69)$ and normal mesothelial cells (MCs; $\mathrm{n}=4$ ). Only the expression of the IL34 gene was significantly higher in MPM cells compared with MC (figure 3A,B). In this collection of primary tumor cells, CSF1 and IL34 mRNA expressions were not associated with histologic subtypes of MPM (see online supplementary figure S5). Higher expression of IL34 was also observed in a collection of MPM cell lines $(n=30)$, established from PE of patients with MPM, compared with primary mesothelial cells $(n=7)$ or other neoplasia cell lines $(n=7)$ also established from PE of patients (see online supplementary figure S6). Interestingly, we observed that overexpression of IL34 in primary MPM cells was strongly associated with genetic alterations, consisting mainly in biallelic deletions of the $C D K N 2 A$ gene and weakly with mutations in the NF2 gene, whereas CSF1 expression was independent of the mutational status (figure 3C-F). No other significant association was found between IL34 or CSF1 expression and genetic alterations in BAP1, LATS2 


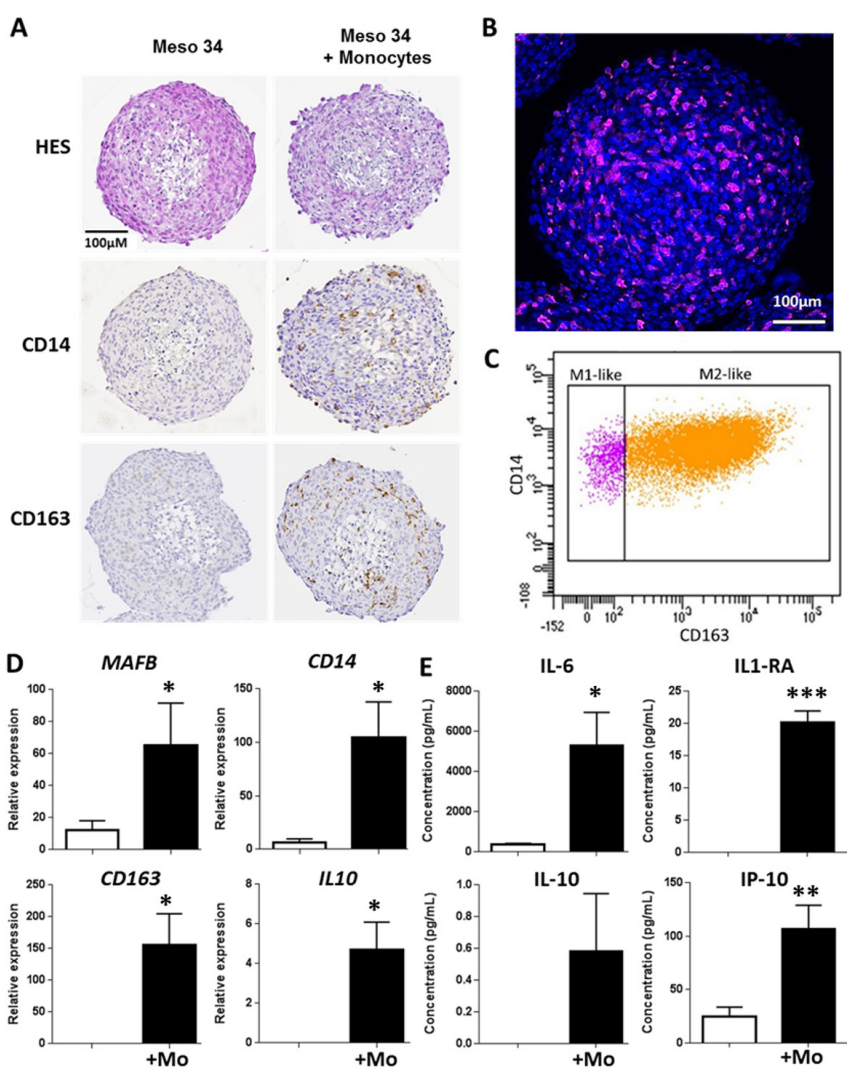

Figure 4 Evaluation of malignant pleural mesothelioma (MPM) cell capacity to drive monocyte differentiation into M2-like macrophages using a multicellular tumor spheroid (MCTS) model. Meso 34 cells were cultured with or without $30 \%$ of monocytes (Mo) in low adherence conditions for 3 days. (A) Macrophage phenotype was studied using immunohistochemistry with CD14 or CD163 labeling. To confirm the presence of macrophages, (B) MCTSs were labeled with an anti-CD163 antibody coupled to Alexa-Fluor 647 (purple), Hoechst for nuclei staining (blue) and observed using confocal microscopy. (C) Representative analysis of three independent flow cytometry experiments showing CD14 and CD163 expression on HLA-DR+ and CD14+ cells in MCTS. (D) Expressions of MAFB, CD14, CD163 and IL10 mRNA were measured using quantitative realtime PCR. Results are means \pm SEM of six independent experiments. ${ }^{\star} p<0.05$; ${ }^{\star *} p<0.01$. (E) Impact of the presence of macrophages in MCTS was determined by measuring the levels of IL-6, IL-1RA, IL-10 and IP-10 (CXCL10) in MCTS culture supernatants. Results are means \pm SEM of six independent experiments. ${ }^{* *} p<0.01$.

or TP53 genes or with $\mathrm{C} 1 / \mathrm{C} 2$ subtypes of the molecular classification. $^{12}$

\section{Evaluation of the capacity of MPM cells to induce the differentiation of monocytes into M2 macrophages}

With the objective to be closer to the physiopathological situation, we developed models of cell culture in three dimensions, namely, multicellular tumor spheroids (MCTSs). Two MPM cell lines with spontaneous capacity to form MCTS were used. The characteristics of these cell lines are provided in online supplementary figure S7 and online supplementary table S2. Figure 4A shows that coculture of Meso 34 with monocytes led to the formation of Mo-MCTS that contain CD14+ and CD163+ cells. In MCTS constituted only of Meso 34 cells, there was neither CD14 nor CD163 labeling. Using confocal microscopy, we observed CD163-positive cells (pink) inside the Mo-MCTS after a transparisation procedure (figure 4B). In order to improve the characterization of macrophages obtained, we used flow cytometry. MCTSs were dissociated and cells were labeled with antibodies anti-CCR2, anti-HLA-DR, anti-CD14 and anti-CD163 to determine the M1-like or M2-like phenotype. Monocytes obtained by elutriation were CCR2 high, CD14 high, CD163 low and HLA-DR mild (online supplementary figure S8A-D). As controls, monocytes from elutriation were incubated with GM-CSF to obtain M1-like macrophages, characterized by a CCR2 low, CD14 low, CD163 low and HLA-DR mild expression, or with M-CSF to obtain M2-like macrophages, characterized by a CCR2 low, CD14 high, CD163 high and HLA-DR mild expression (see online supplementary figure S8). In MCTS, myeloid cells expressed HLA-DR and CD14, whereas mesothelioma cells did not express HLA-DR and expressed low level of CD14 (see online supplementary figure S9A). Approximately $85 \%$ of the myeloid cells were CD14 high and CD163 high (figure 4C and online supplementary figure S9B), corresponding probably to M2-like macrophages, and approximately $15 \%$ of the myeloid cells were CD14 mild and CD163 low, corresponding probably to M1-like macrophages (figure $4 \mathrm{C}$ and online supplementary figure S9B). This suggests that in MCTS, monocytes differentiated mainly into 'M2-like' macrophages. To confirm this hypothesis, we measured the mRNA expression of MAFB, CD14, CD163 and IL10. Expressions of all these macrophage markers were higher in MCTS made of Meso 34+ monocytes than in MCTS containing only Meso 34 cells (figure 4D). No major change in the mRNA transcription of the modulators of the immune response PDL-1, PDL-2, GITRL and OX40L was observed in the presence of macrophages (see online supplementary figure S10). Similar results were obtained with Meso 13 (see online supplementary figure S9C and D, figure S11). Analysis of the cytokines secreted by MCTS in culture supernatants showed that in the presence of monocytes, levels of IL-6, IL1-RA, IL-10 and IP-10 (figure $4 \mathrm{E}$ ), tumor necrosis factor alpha (TNF $\alpha$ ) and IL-1 $\beta$ (see online supplementary figure S12) were higher than in condition with Meso 34 alone. This set of cytokines allowed us to discriminate M1 from M2 macrophages, as shown in online supplementary figure S12. The profile of cytokines expressed in MCTS supernatants was similar to the one of M2 macrophages: absence of IL-12, presence of high amount of IL-6, IP-10 and presence of IL-10. Altogether, these results suggest that the coculture of MPM cells with monocytes as MCTS led to the differentiation of monocytes into 'M2-like' macrophages.

We evaluated the involvement of the CSF-1R pathway in this process by adding a CSF-1R inhibitor, GW2580, during the formation of the MCTS. Figure 5A shows that treatment with GW2580 reduced the expression of 
A

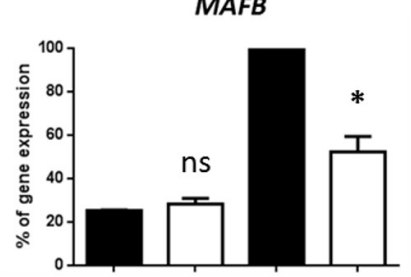

CD163

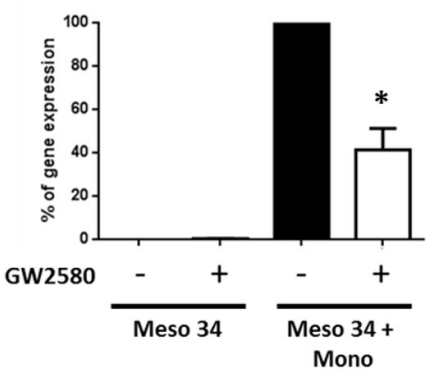

B

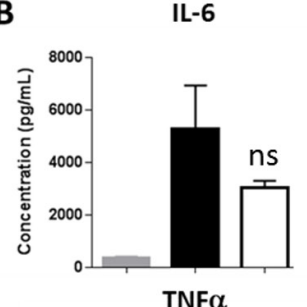

TNF $\alpha$

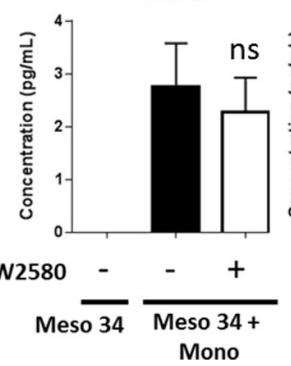

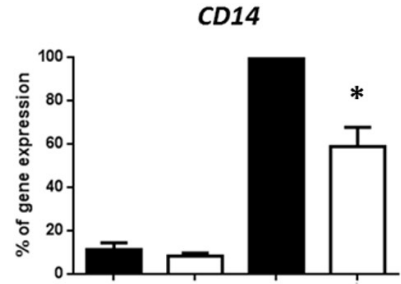

IL10

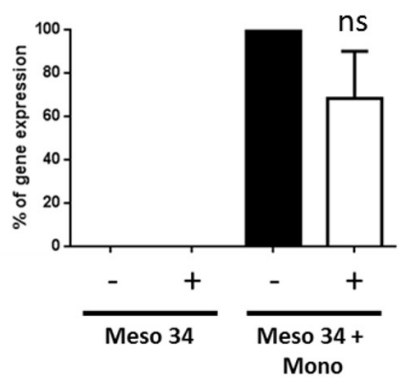

IL1-RA

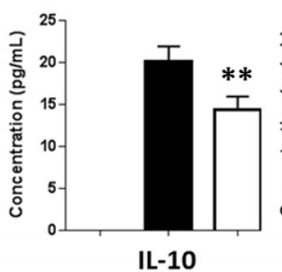

IL-10

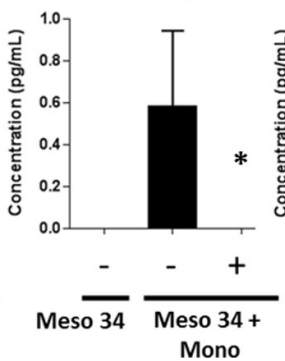

IL-1 $\beta$
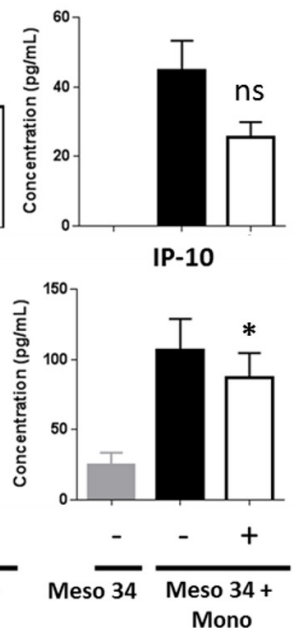

Figure 5 Effect of CSF-1R inhibition on macrophages in multicellular tumor spheroid (MCTS). Meso 34 cells were cultured with or without $30 \%$ of monocytes (Mono) in low adherence conditions for 3 days in the presence or not of $1 \mu \mathrm{M}$ GW2580. (A) Expressions of MAFB, CD14, CD163 and IL10 mRNA were measured using quantitative real-time PCR. Results are means \pm SEM of three independent experiments. ${ }^{*} \mathrm{p}<0.05$; ns, non-significant. (B) Levels of interleukin (IL)-6, IL-1RA, IL-1 $\beta$, tumor necrosis factor alpha (TNF $\alpha$ ), IL-10 and IP-10 (CXCL10) in MCTS culture supernatants. Results are means \pm SEM of six independent experiments. ${ }^{*} p<0.05$; ${ }^{* *} \mathrm{p}<0.01$; ns, non-significant.

macrophage markers MAFB, CD14,CD163 and IL10 by approximately $50 \%$. These observations were associated with a decrease of the levels of IL-6, IL-1RA, IL-1 $\beta$, TNF $\alpha$, IL-10 and IP-10 in the culture supernatants of MCTS (figure 5B and see online supplementary figure S13).

Impact of the presence of macrophages on the cytotoxic activity of a tumor antigen-specific CD8+ $T$ cell clone

In order to evaluate the immunological impact of the presence of macrophages in MCTS, we measured the cytotoxic activity of a tumor antigen-specific $\mathrm{CD}^{+} \mathrm{T}$ cell
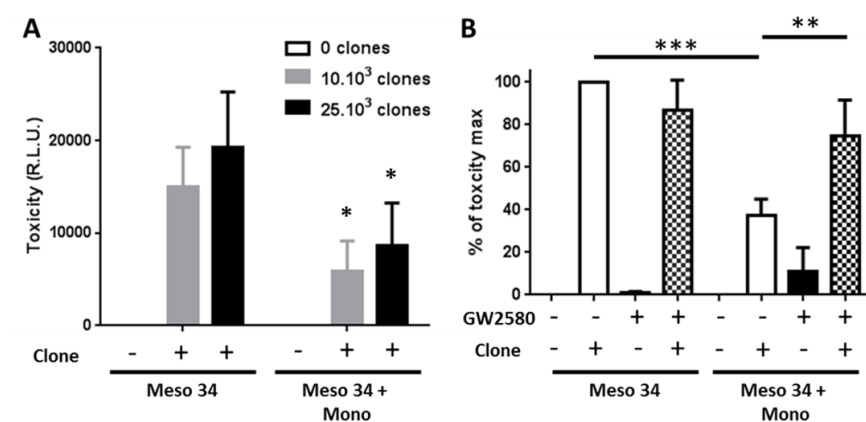

Figure 6 Effect of macrophage and CSF-1R inhibition on the specific CD8 T cell clone cytotoxic activity against MPM cells. Meso 34 NanoLuc cells were cultured with or without monocytes (Mono) in low adherence conditions. After 3 days, a MUC1-specific CD8 T cell clone was added for 24 hours. Then, supernatants were collected and NanoLuc activity was measured to determine cell lysis. (A) Effect of the presence of macrophages on the cytotoxic activity of the MUC1-specific $T$ cell clone. Results are means \pm SEM of four independent experiments. Meso34 vs Meso34+Mono: ${ }^{*} p<0.05$. (B) Impact of the inhibition of CSF-1R, using GW2580 $(1 \mu \mathrm{M})$, on the T cell clone cytotoxic activity. ${ }^{* *} p<0.01 ;{ }^{* \star *} p<0.001$. CSF-1R, colony stimulatingfactor-1 Receptor; MPM, malignant pleural mesothelioma; RLU, relative light units.

clone toward MPM cells. We used an HLA-A2*0201/ MUC1 (950-958)-specific T cell clone that recognizes the HLA-A2+MUC1+Meso 34 MPM cell line. ${ }^{17}$ The coculture of Meso 34 NanoLuc MCTS with the $\mathrm{CD} 8^{+} \mathrm{T}$ cell clone induced a release of luciferase activity in the culture supernatants of MCTS demonstrating the lysis of the MPM cells (figure 6A). This cytotoxicity was correlated with the number of specific $\mathrm{CD}^{+} \mathrm{T}$ cell used. In the presence of macrophages in the MCTS, the activity of the $\mathrm{CD}^{+} \mathrm{T}$ cell clone was reduced by approximately $60 \%$ (figure 6A). Inhibition of CSF-1R by GW2580 restored significantly the cytotoxicity of the CD8 ${ }^{+} \mathrm{T}$ cells from $40 \%$ up to $75 \%$ (figure $6 \mathrm{~B}$ ).

\section{DISCUSSION}

MPM is a rare and aggressive cancer with few treatments available. A better understanding of the tumor environment could lead to novel therapeutic strategies by identifying new targets. Recently, it was shown that the presence of macrophages, and particularly M2-like, CD163+ macrophages, was associated with a lower survival of patients. ${ }^{3}$ We and others previously showed that PEs from patients with MPM led to the differentiation of monocytes into M2-like macrophages. ${ }^{719}$ We observed in particular in a limited number of samples the presence of M-CSF, wellknown to be implicated in this process. In this study, we aimed at going deeper into the characterization of the expression of factors which could lead to macrophage formation. We focused our study on M-CSF and IL-34, two ligands of CSF-1R. We showed, using our cohort of pleural effusions from patients, that IL-34, but not M-CSF, was specifically expressed in malignant $\mathrm{PE}$, including MPM PE, compared with benign PE. High expressions of 
M-CSF and presence of IL-34 in PE were associated with a shorter survival of patients with MPM. At the mRNA level in MPM tumors, CSFI expression, but not IL34, was correlated with the expression of M2-like macrophage markers. In primary MPM cell lines, but also in MPM cell lines established from PE of patients, we observed an overexpression of IL34 compared with normal mesothelial cells, particularly in MPM cells with an alteration of CDKN2A. Using MCTS three-dimensional (3D) coculture models, we demonstrated that MPM cells were able, by themselves, to induce the differentiation of monocytes into M2-like macrophages through activation of the CSF-1R pathway. In this model, we observed that M2-like macrophages present in MCTS led to a strong reduction of the cytotoxic activity of a tumor antigen-specific human $\mathrm{CD}^{+} \mathrm{T}$ cell clone toward MPM cells. The use of a CSF-1R inhibitor restored the cytotoxic activity of this $\mathrm{CD}^{+} \mathrm{T}$ cell clone.

M-CSF and IL-34 are two cytokines well-known to be involved in macrophage differentiation through their binding to CSF-1R. ${ }^{8}$ M-CSF is implicated in survival and differentiation of monocytes but also in tissue repair and inflammation. In several cancers, it was observed that the levels of M-CSF were elevated and associated with a poor prognosis. ${ }^{20}$ In PE, M-CSF was not a biomarker of malignancy. Indeed, levels of M-CSF were similar in malignant $\mathrm{PE}$ and BPE, thus suggesting that the presence of this cytokine could be related to the inflammatory environment as previously observed. ${ }^{21}$ An association between high M-CSF levels and shorter patient survival was nevertheless observed. A possible explanation could be the induction of M2-like macrophages by M-CSF supported by the correlation of $\mathrm{CSF} 1$ expression with $\mathrm{CD} 163, \mathrm{CD} 14$ and ILIO in MPM tumors. Indeed, the presence of M2 macrophages was already associated with a worse prognosis in $\mathrm{MPM}^{35}$ and we already demonstrated the implication of the CSF-1R pathway in their formation induced by MPM PE. ${ }^{7}$

IL-34, a more recently identified cytokine ${ }^{22}$ is also involved in monocyte survival and differentiation but the situation regarding MPM was different compared with M-CSF. Indeed, significantly higher levels of this cytokine were measured in malignant PE compared with BPE. However, it is important to note that more than $50 \%$ of PE were negative for IL-34, whereas all the PE were positive for M-CSF. Recent studies have evidenced the expression of IL-34 in several cancers and a correlation between a high expression of IL-34 and shorter survival of patients. ${ }^{23}{ }^{24}$ A negative prognostic value of IL-34 in MPM was also observed in our study when comparing positive and negative PE, and IL-34 was an independent factor contrary to M-CSF when taking into account histology. This suggests a strong involvement of this cytokine in MPM development and in the outcome of the disease. The absence of correlation between IL34 expression and M2-like macrophage markers, observed in tumors, suggests an action of this cytokine through another mechanism beside macrophage induction. Recently, a similar situation was reported in breast cancer. ${ }^{25}$ An autocrine proliferative action of IL-34 on tumor cells was described in liver cancer cell lines ${ }^{26}$ and also in MPM cell lines, ${ }^{27}$ which could explain the poor prognostic value of IL-34.

We observed a higher expression of IL34 in MPM cells with a biallelic deletion of $C D K N 2 A$. The link with the NF2 mutation status was weaker and could be due to the fact that most of NF2-mutated MPM primary cell lines also show a $C D K N 2 A$ biallelic deletion. In the chromosomal region 9p21.3, frequently deleted in MPM, the locus of the gene coding for miR31 can also be found close to the $C D K N 2 A$ and $C D K N 2 B$ loci. Consequently, the MIR31 gene is frequently co-deleted with the $C D K N 2 A$ gene. Interestingly, miR-31 targets $I L 34$ to reduce its expression. ${ }^{28}$ Thus, it would be interesting to study miR-31 expression in our cells to evaluate the possible relation with IL34 expression and with the deletion of CDKN2A. miR-31 is usually dysregulated in cancer; however, it could act either as a tumor suppressor or as an oncogene. ${ }^{29}$ In MPM, the situation is still controversial. Some articles demonstrated a positive impact of the expression of miR-31, as shown by an association with a better prognosis and a decrease of the oncogenic properties of MPM cells ${ }^{30}$ whereas others observed an association with a poor prognosis ${ }^{31}$ and a positive correlation with sensitivity to chemotherapy. ${ }^{32}$

We previously demonstrated the involvement of the CSF-1R pathway in the formation of M2-like macrophages induced by MPM PE. ${ }^{7}$ In this study, using MCTS containing MPM cells and monocytes (Mo-MCTS), we demonstrated the direct involvement of MPM cells in this process via the CSF-1R pathway using a specific inhibitor. As observed in tumors, macrophages obtained in Mo-MCTS expressed CD163, CD14, IL10 and MAFB. At the cytokine level, supernatants from Mo-MCTS presented some similarities and PE from MPM patients with high IL- 6 concentrations and the presence of IL-10 and TNF $\alpha .{ }^{19}$ Moreover, as observed in MPM tumors, high expression of CSF1 was associated with high expression of CD163. Indeed, Meso 13 expressed higher level of CSF1 and of M-CSF than Meso 34 and Mo-MCTS constituted with Meso 13 expressed more CD163 than those constituted with Meso34 (see online supplementary figure S7).

In MCTS, the cytokine profile obtained in the supernatant in the presence of monocytes was similar to the one of M2 macrophages. The high levels of TNF $\alpha$ and IL-1 $\beta$ in MCTS supernatants may be due to the presence of a small fraction of M1-like macrophages that we observed, thanks to the characterization of macrophages by flow cytometry after dissociation of MCTS. The expression profile of mRNA markers and cytokines suggested that macrophages in Mo-MCTS displayed a M2-like phenotype with potent immunosuppressive properties. ${ }^{8}$ The inhibition of the cytotoxic activity of a $\mathrm{CD} 8^{+} \mathrm{T}$ cell clone by Mo-MCTS, as observed in cytotoxic assays, provides new pieces of evidence which reinforced our hypothesis. Beyond mechanistic results, these Mo-MCTSs could constitute a unique model to study MPM cells and monocytes interactions and also for the screening of agents 
with anti-immunosuppressive properties or immunomodulating properties. Indeed, we showed that the use of GW2580, which reduced the formation of M2-like macrophages as demonstrated by the expression of specific cellular markers and cytokines, restored the cytotoxic activity of $\mathrm{CD}^{+} \mathrm{T}$ cells.

The targeting of macrophages represents a growing field of interest in cancer therapy. ${ }^{33}$ In patients with diffuse-type giant cell tumor, blocking of CSF-1R led to a decrease of CSF-1 $\mathrm{R}^{+} \mathrm{CD} 163^{+}$macrophages in tumor tissue associated with an increase of lymphocyte infiltration. ${ }^{34}$ This strategy has already demonstrated its efficacy in a mouse model of mesothelioma using combined immunotherapy. ${ }^{35}$ Thanks to our Mo-MCTS model, which represents an intermediate and simple model to replace some in vivo experiments and to develop new antiimmunosuppressive agents, we highlighted the efficacy of blocking CSF-1R to inhibit M2 macrophage differentiation from monocyte and to restore human $\mathrm{CD}^{+}$effector $\mathrm{T}$ cell function. Moreover, inhibition of CSF-1R, rather than blocking one cytokine, could also be beneficial to inhibit the deleterious effect of IL-34 in MPM suggested by patient survival analysis and a previous study. ${ }^{27}$

\section{CONCLUSIONS}

In summary, this work shows that M-CSF/IL-34/CSF-1R pathway is involved in MPM pathology. IL-34 could be a new interesting prognostic biomarker for MPM, but additional studies on larger cohort are required to confirm this observation. Our models of MCTS demonstrate the direct capacity of MPM cells to induce immunoregulatory macrophage formation through stimulation of the CSF-1R pathway. Finally, we confirmed in our MCTS coculture models the immunosuppressive nature of CSF-1R mediated differentiation of macrophages directly on the cytotoxic activity of tumor antigen-specific CD8+ T cells. Thus, inhibition of CSF-1R pathway could be a promising approach to favor the antitumor $\mathrm{T}$ cell response in patients with MPM.

\section{Author affiliations}

${ }^{1}$ Université de Nantes, CNRS, INSERM, CRCINA, F-44000 Nantes, France

${ }^{2}$ Université d'Angers, INSERM, CRCINA, F-49000 Angers, France

${ }^{3}$ Ludwig Center for Cancer Research, University of Lausanne, Lausanne, Switzerland ${ }^{4}$ Flow Cytometry Core Facility, School of Life Sciences, Ecole Polytechnique Fédérale de Lausanne (EPFL), Lausanne, Switzerland

${ }^{5}$ Centre de Recherche des Cordeliers, Inserm, Sorbonne Université, Université de Paris, Functional Genomics of Solid Tumors, F-75006, Paris, France

${ }^{6}$ Service d'Oncologie Médicale Thoracique et Digestive, Hopital Nord Laennec, Nantes, Pays de la Loire, France

${ }^{7} \mathrm{CHU}$ Angers, Laboratoire d'Immunologie et Allergologie, F-49000 Angers, France

${ }^{8} \mathrm{CHU}$ de Nantes, oncologie thoracique et oncologie digestive, 5, allée de l'île Gloriette, 44093 Nantes, France

Acknowledgements This work was supported by INSERM, CNRS, the 'Institut de recherche en santé respiratoire des Pays de la Loire', the 'Université de Nantes', the 'Région des Pays de la Loire', the 'Ligue Contre le Cancer' (committees of Morbihan, Sarthe, Vendée et Loire-Atlantique and lle de France committee), ARSMES044, Hadassah France, Chancellerie des Universités de Paris (Legs POIX), the National Research Agency under the Programme d'Investissements d'Avenir
(ANR-16-IDEX-0007) and the Pays de la Loire Region research programme. The authors thank the cluster LUNG innOvatiOn (LUNG 02) for logistic support, the Cytometry Facility « Cytocell » from Nantes for their expert technical assistance, the MicroPiCell core facility (SFR François Bonamy) for microscopy analyses, immunohistology, and the DTC core facility (CIC-biothérapies Nantes) for human monocyte purification.

Contributors $\mathrm{CB}$ and MG: responsible for study design and execution, data collection, data analysis and manuscript preparation. TB, TB, SMd, JT, CM, SD: responsible for study execution and data collection. A-LC, LC and JB: Responsible for sample collection. YD, J-FF, NB and DJ: participation to data analysis and manuscript preparation.

Funding This work was supported by INSERM, CNRS, the 'Institut de recherche en santé respiratoire des Pays de la Loire', the 'Ligue Contre le Cancer' (committees of Morbihan, Sarthe, Vendée et Loire-Atlantique and lle de France committee), Hadassah France, Chancellerie des Universités de Paris (Legs POIX), and ARSMES044.

Competing interests None declared.

Patient consent for publication Not required.

Ethics approval All recruited patients gave signed, informed consent. All the collected samples and the associated clinical information were registered in database (DC-2011-1399 and DC-2013-1963) validated by the French research ministry.

Provenance and peer review Not commissioned; externally peer reviewed.

Data availability statement The datasets used and analyzed during the current study are available from the corresponding author

Open access This is an open access article distributed in accordance with the Creative Commons Attribution Non Commercial (CC BY-NC 4.0) license, which permits others to distribute, remix, adapt, build upon this work non-commercially, and license their derivative works on different terms, provided the original work is properly cited, appropriate credit is given, any changes made indicated, and the use is non-commercial. See http://creativecommons.org/licenses/by-nc/4.0/.

\section{ORCID iD}

Christophe Blanquart http://orcid.org/0000-0002-0917-3747

\section{REFERENCES}

1 Vogelzang NJ, Rusthoven JJ, Symanowski J, et al. Phase III study of pemetrexed in combination with cisplatin versus cisplatin alone in patients with malignant pleural mesothelioma. $J$ Clin Oncol 2003;21:2636-44.

2 Burt BM, Rodig SJ, Tilleman TR, et al. Circulating and tumorinfiltrating myeloid cells predict survival in human pleural mesothelioma. Cancer 2011;117:5234-44.

3 Ujiie H, Kadota K, Nitadori J-I, et al. The tumoral and stromal immune microenvironment in malignant pleural mesothelioma: a comprehensive analysis reveals prognostic immune markers. Oncoimmunology 2015;4:e1009285.

4 Mantovani A, Marchesi F, Malesci A, et al. Tumour-Associated macrophages as treatment targets in oncology. Nat Rev Clin Oncol 2017;14:399-416.

5 Cornelissen R, Lievense LA, Maat AP, et al. Ratio of intratumoral macrophage phenotypes is a prognostic factor in epithelioid malignant pleural mesothelioma. PLoS One 2014;9:e106742.

6 Cornelissen R, Lievense LA, Robertus J-L, et al. Intratumoral macrophage phenotype and CD8+ T lymphocytes as potential tools to predict local tumor outgrowth at the intervention site in malignant pleural mesothelioma. Lung Cancer 2015;88:332-7.

7 Chéné A-L, d'Almeida S, Blondy T, et al. Pleural effusions from patients with mesothelioma induce recruitment of monocytes and their differentiation into M2 macrophages. J Thorac Oncol 2016;11:1765-73.

8 Jeannin P, Paolini L, Adam C, et al. The roles of CSFS on the functional polarization of tumor-associated macrophages. Febs $J$ 2018;285:680-99.

9 Baghdadi M, Endo H, Tanaka Y, et al. Interleukin 34, from pathogenesis to clinical applications. Cytokine 2017;99:139-47.

10 Foucher ED, Blanchard S, Preisser L, et al. IL-34- and M-CSFinduced macrophages switch memory T cells into Th17 cells via membrane IL-1 $\alpha$. Eur J Immunol 2015;45:1092-102.

11 Gueugnon F, Leclercq S, Blanquart C, et al. Identification of novel markers for the diagnosis of malignant pleural mesothelioma. Am J Pathol 2011;178:1033-42. 
12 de Reyniès A, Jaurand M-C, Renier A, et al. Molecular classification of malignant pleural mesothelioma: identification of a poor prognosis subgroup linked to the epithelial-to-mesenchymal transition. Clin Cancer Res 2014;20:1323-34.

13 Tranchant R, Quetel L, Tallet A, et al. Co-occurring Mutations of Tumor Suppressor Genes, LATS2 and NF2, in Malignant Pleural Mesothelioma. Clin Cancer Res 2017;23:3191-202.

14 Smeele P, d'Almeida SM, Meiller C, et al. Brain-Derived neurotrophic factor, a new soluble biomarker for malignant pleural mesothelioma involved in angiogenesis. Mol Cancer 2018;17:148.

15 Blum Y, Meiller C, Quetel L, et al. Dissecting heterogeneity in malignant pleural mesothelioma through histo-molecular gradients for clinical applications. Nat Commun 2019;10:1333.

16 Coulais D, Panterne C, Fonteneau J-F, et al. Purification of circulating plasmacytoid dendritic cells using counterflow centrifugal elutriation and immunomagnetic beads. Cytotherapy 2012;14:887-96.

17 Roulois D, Vignard V, Gueugnon F, et al. Recognition of pleural mesothelioma by mucin-1(950-958)/human leukocyte antigen A*0201-specific CD8+ T-cells. Eur Respir J 2011;38:1117-26.

18 Becht E, de Reyniès A, Giraldo NA, et al. Immune and stromal classification of colorectal cancer is associated with molecular subtypes and relevant for precision immunotherapy. Clin Cancer Res 2016;22:4057-66.

19 Lievense LA, Cornelissen R, Bezemer K, et al. Pleural effusion of patients with malignant mesothelioma induces macrophagemediated T cell suppression. J Thorac Oncol 2016;11:1755-64.

20 Laoui D, Van Overmeire E, De Baetselier P, et al. Functional relationship between tumor-associated macrophages and macrophage colony-stimulating factor as contributors to cancer progression. Front Immunol 2014;5:489.

21 Hamilton JA, Cook AD, Tak PP. Anti-colony-stimulating factor therapies for inflammatory and autoimmune diseases. Nat Rev Drug Discov 2016;16:53-70.

22 Lin H, Lee E, Hestir K, et al. Discovery of a cytokine and its receptor by functional screening of the extracellular proteome. Science 2008;320:807-11.

23 Wang B, Xu W, Tan M, et al. Integrative genomic analyses of a novel cytokine, interleukin-34 and its potential role in cancer prediction. Int J Mol Med 2015;35:92-102.
24 Zhou S-L, Hu Z-Q, Zhou Z-J, et al. miR-28-5p-IL-34-macrophage feedback loop modulates hepatocellular carcinoma metastasis. Hepatology 2016;63:1560-75.

25 Zins K, Heller G, Mayerhofer M, et al. Differential prognostic impact of interleukin-34 mRNA expression and infiltrating immune cell composition in intrinsic breast cancer subtypes. Oncotarget 2018;9:23126-48.

26 Baghdadi M, Wada H, Nakanishi S, et al. Chemotherapy-Induced IL34 enhances immunosuppression by tumor-associated macrophages and mediates survival of chemoresistant lung cancer cells. Cancer Res 2016;76:6030-42.

27 Cioce M, Canino C, Goparaju C, et al. Autocrine CSF-1R signaling drives mesothelioma chemoresistance via Akt activation. Cell Death Dis 2014;5:e1167.

28 Li M, Dong Y, Chen Z, et al. Microrna-31 negatively regulates interleukin-34 expression in vitro. Immunol Invest 2019;48:1-11.

29 Truini A, Coco S, Genova C, et al. Prognostic and therapeutic implications of microRNA in malignant pleural mesothelioma. Microrna 2016;5:12-18.

30 Ivanov SV, Goparaju CMV, Lopez P, et al. Pro-Tumorigenic effects of miR-31 loss in mesothelioma. J Biol Chem 2010;285:22809-17.

31 Matsumoto S, Nabeshima K, Hamasaki M, et al. Upregulation of microRNA-31 associates with a poor prognosis of malignant pleural mesothelioma with sarcomatoid component. Med Oncol 2014;31:303.

32 Moody HL, Lind MJ, Maher SG. Microrna-31 regulates chemosensitivity in malignant pleural mesothelioma. Mol Ther Nucleic Acids 2017;8:317-29.

33 Brown JM, Recht L, Strober S. The promise of targeting macrophages in cancer therapy. Clin Cancer Res 2017;23:3241-50.

34 Ries $\mathrm{CH}$, Cannarile MA, Hoves S, et al. Targeting tumor-associated macrophages with anti-CSF-1R antibody reveals a strategy for cancer therapy. Cancer Cell 2014;25:846-59.

35 Dammeijer F, Lievense LA, Kaijen-Lambers ME, et al. Depletion of tumor-associated macrophages with a CSF-1R kinase inhibitor enhances antitumor immunity and survival induced by DC immunotherapy. Cancer Immunol Res 2017;5:535-46. 